Supplement of Biogeosciences, 15, 2289-2307, 2018

https://doi.org/10.5194/bg-15-2289-2018-supplement

(C) Author(s) 2018. This work is distributed under

the Creative Commons Attribution 4.0 License.

(c) (1)

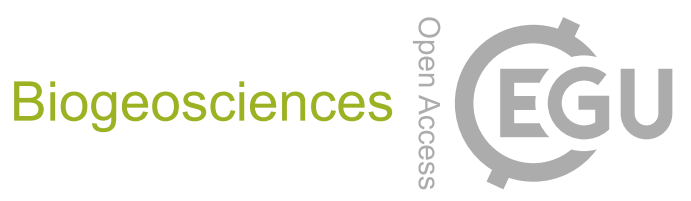

Supplement of

\title{
Particulate barium tracing of significant mesopelagic carbon reminerali- sation in the North Atlantic
}

Nolwenn Lemaitre et al.

Correspondence to: Nolwenn Lemaitre (nolwenn.lemaitre@erdw.ethz.ch)

The copyright of individual parts of the supplement might differ from the CC BY 4.0 License. 


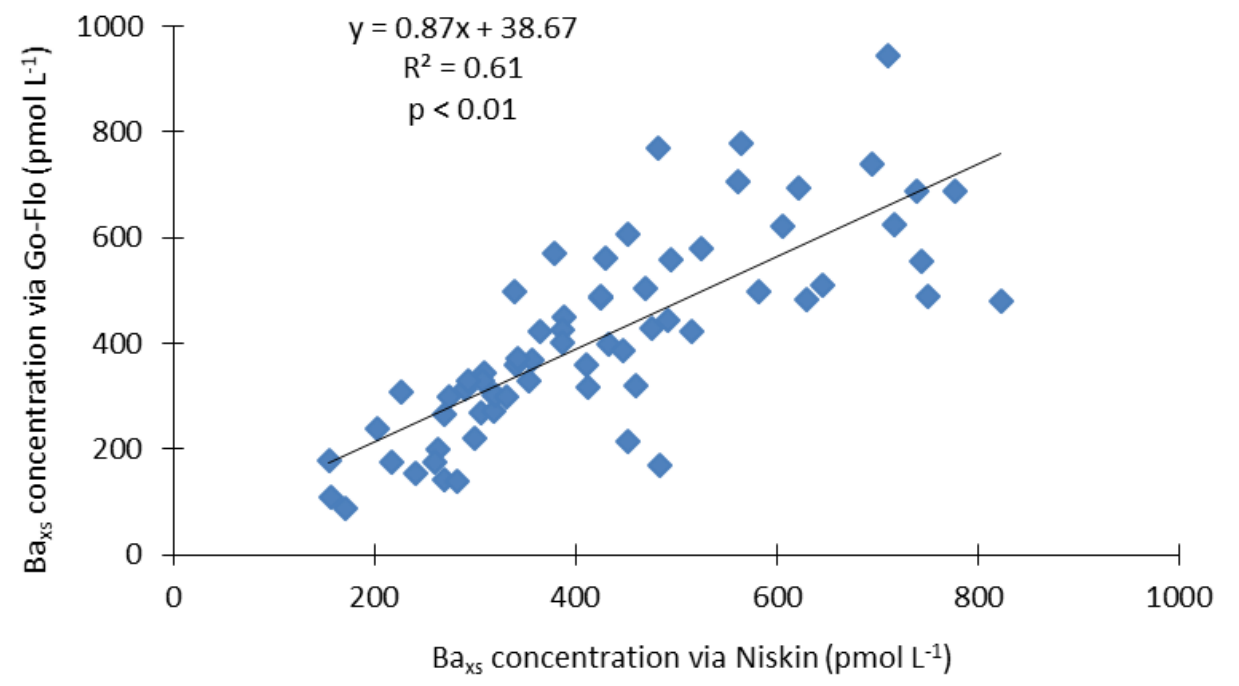

2 Figure S1: Comparison between the Baxs concentrations obtained on samples collected by two different sampling and 3 analytical methods (Niskin, $0.40 \mu \mathrm{m}$ polycarbonate filters; and Go-Flo systems, paired $0.45 \mu \mathrm{m}$ polyethersulfone and $5 \mu \mathrm{m}$ 4 mixed ester cellulose filters, see Gourain et al., 2018; this issue) for the 100-1000 m layer. See text for details. 
5 Table S1: Particulate biogenic barium (Baxs; in pmol $\left.\mathbf{L}^{-1}\right)$ during GEOVIDE, obtained on samples collected from Niskin

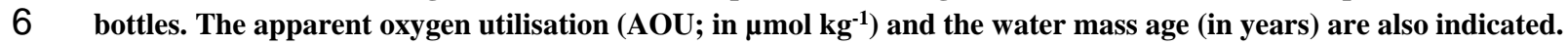

\begin{tabular}{|c|c|c|c|c|c|c|c|c|c|c|c|c|c|c|c|c|c|}
\hline \multicolumn{6}{|c|}{ Station $1\left(40.3^{\circ} \mathrm{N} /-10.0^{\circ} \mathrm{E}\right)$} & \multicolumn{6}{|c|}{ Station $13\left(41.4^{\circ} \mathrm{N} /-13.9^{\circ} \mathrm{E}\right)$} & \multicolumn{6}{|c|}{ Station $21\left(46.5^{\circ} \mathrm{N} /-19.7^{\circ} \mathrm{E}\right)$} \\
\hline \multirow{2}{*}{$\begin{array}{l}\text { Depth } \\
(\mathrm{m}) \\
20\end{array}$} & \multicolumn{3}{|c|}{$\begin{array}{c}\mathrm{Ba}_{\mathrm{xs}} \\
\left(\mathrm{pmol} \mathrm{L}{ }^{-1}\right)\end{array}$} & \multirow[t]{2}{*}{$\begin{array}{l}\mathrm{AOU} \\
(\mu \mathrm{mol} \\
\left.\mathrm{kg}^{-1}\right)\end{array}$} & \multirow[t]{2}{*}{$\begin{array}{c}\text { Water } \\
\text { mass age } \\
\text { (years) }\end{array}$} & \multirow{2}{*}{$\begin{array}{l}\text { Depth } \\
(\mathrm{m}) \\
20\end{array}$} & \multicolumn{3}{|c|}{$\begin{array}{c}\mathrm{Ba}_{\mathrm{xs}} \\
\left(\mathrm{pmol} \mathrm{L}{ }^{-1}\right)\end{array}$} & \multirow[t]{2}{*}{$\begin{array}{c}\mathrm{AOU} \\
(\mu \mathrm{mol} \\
\left.\mathrm{kg}^{-1}\right)\end{array}$} & $\begin{array}{l}\text { Water } \\
\text { mass age } \\
\text { (years) }\end{array}$ & \multirow{2}{*}{$\begin{array}{l}\text { Depth } \\
(\mathrm{m}) \\
10\end{array}$} & \multicolumn{3}{|c|}{$\begin{array}{c}\mathrm{Ba}_{\mathrm{xs}} \\
\left(\mathrm{pmol} \mathrm{L}{ }^{-1}\right)\end{array}$} & \multirow[t]{2}{*}{$\begin{array}{c}\mathrm{AOU} \\
(\mu \mathrm{mol} \\
\left.\mathrm{kg}^{-1}\right)\end{array}$} & $\begin{array}{c}\text { Water } \\
\text { mass age } \\
\text { (years) }\end{array}$ \\
\hline & 126 & \pm & 19 & & & & 239 & \pm & 53 & & & & 207 & \pm & 36 & & \\
\hline 40 & 195 & \pm & 13 & & & 40 & 218 & \pm & 38 & & & 20 & 566 & \pm & 113 & & \\
\hline 60 & 214 & \pm & 17 & & & 60 & 177 & \pm & 42 & & & 40 & 276 & \pm & 45 & & \\
\hline 79 & 363 & \pm & 35 & & & 78 & 242 & \pm & 48 & & & 60 & 181 & \pm & 35 & & \\
\hline 98 & 412 & \pm & 80 & 19 & 6.4 & 100 & 274 & \pm & 50 & 10 & 3.1 & 76 & 483 & \pm & 79 & & \\
\hline 119 & 291 & \pm & 67 & & & 120 & 306 & \pm & 50 & & & 100 & 453 & \pm & 73 & 14 & 2.1 \\
\hline 139 & 288 & \pm & 64 & & & 140 & 379 & \pm & 71 & & & 120 & 467 & \pm & 66 & & \\
\hline 160 & 241 & \pm & 54 & 22 & 6.6 & 160 & 449 & \pm & 70 & & & 140 & 502 & \pm & 74 & 14 & 1.6 \\
\hline 200 & 340 & \pm & 88 & 30 & 8.6 & 200 & 425 & \pm & 64 & 14 & 2.9 & 160 & 338 & \pm & 56 & & \\
\hline 249 & 470 & \pm & 111 & & & 250 & 496 & \pm & 87 & & & 200 & 340 & \pm & 51 & 15 & 2.7 \\
\hline 299 & 310 & \pm & 101 & 43 & 16 & 300 & 582 & \pm & 90 & 31 & 9.0 & 300 & 491 & \pm & 76 & 17 & 4.8 \\
\hline 399 & 263 & \pm & 70 & 54 & 25 & 400 & 961 & \pm & 147 & 17 & 4.6 & 400 & 379 & \pm & 57 & 34 & 13 \\
\hline 499 & 286 & \pm & 86 & 72 & 37 & 500 & 357 & \pm & 62 & 34 & 13 & 500 & 524 & \pm & 91 & 17 & 7.6 \\
\hline 597 & 483 & \pm & 103 & 79 & 43 & 600 & 319 & \pm & 61 & 51 & 22 & 600 & 424 & \pm & 69 & 25 & 12 \\
\hline 702 & 156 & \pm & 95 & 81 & 47 & 700 & 305 & \pm & 54 & 79 & 38 & 700 & 364 & \pm & 59 & 79 & 38 \\
\hline 800 & 159 & \pm & 62 & 82 & 50 & 800 & 259 & \pm & 51 & 89 & 48 & 800 & 308 & \pm & 48 & 94 & 49 \\
\hline 1000 & 171 & \pm & 62 & 83 & 58 & 1000 & 270 & \pm & 49 & 88 & 59 & 1000 & 318 & \pm & 62 & 85 & 52 \\
\hline 1505 & 157 & \pm & 62 & & & 1501 & 172 & \pm & 43 & 69 & 0.5 & 1200 & 212 & \pm & 40 & & \\
\hline
\end{tabular}

\begin{tabular}{|c|c|c|c|c|c|c|c|c|c|c|c|c|c|c|c|c|c|}
\hline \multicolumn{6}{|c|}{ Station $26\left(50.3^{\circ} \mathrm{N} /-22.6^{\circ} \mathrm{E}\right)$} & \multicolumn{6}{|c|}{ Station $32\left(55.5^{\circ} \mathrm{N} /-26.7^{\circ} \mathrm{E}\right)$} & \multicolumn{6}{|c|}{ Station $38\left(58.8^{\circ} \mathrm{N} /-31.3^{\circ} \mathrm{E}\right)$} \\
\hline \multirow{2}{*}{$\begin{array}{l}\text { Depth } \\
(\mathrm{m}) \\
20\end{array}$} & \multicolumn{3}{|c|}{$\begin{array}{c}\mathrm{Ba}_{\mathrm{xs}} \\
\left(\mathrm{pmol} \mathrm{L}^{-1}\right)\end{array}$} & \multirow[t]{2}{*}{$\begin{array}{c}\mathrm{AOU} \\
(\mu \mathrm{mol} \\
\left.\mathrm{kg}^{-1}\right)\end{array}$} & \multirow[t]{2}{*}{$\begin{array}{c}\text { Water } \\
\text { mass age } \\
\text { (years) }\end{array}$} & \multirow{2}{*}{$\begin{array}{l}\text { Depth } \\
(\mathrm{m}) \\
10\end{array}$} & \multicolumn{3}{|c|}{$\begin{array}{c}\mathrm{Ba}_{\mathrm{xs}} \\
\left(\mathrm{pmol} \mathrm{L}{ }^{-1}\right)\end{array}$} & \multirow[t]{2}{*}{$\begin{array}{l}\text { AOU } \\
(\mu \mathrm{mol} \\
\left.\mathrm{kg}^{-1}\right)\end{array}$} & $\begin{array}{c}\text { Water } \\
\text { mass age } \\
\text { (years) }\end{array}$ & \multirow{2}{*}{$\begin{array}{l}\text { Depth } \\
(\mathrm{m}) \\
10\end{array}$} & \multicolumn{3}{|c|}{$\begin{array}{c}\mathrm{Ba}_{\mathrm{xs}} \\
\left(\mathrm{pmol} \mathrm{L}^{-1}\right)\end{array}$} & \multirow[t]{2}{*}{$\begin{array}{c}\mathrm{AOU} \\
(\mu \mathrm{mol} \\
\left.\mathrm{kg}^{-1}\right)\end{array}$} & $\begin{array}{l}\text { Water } \\
\text { mass age } \\
\text { (years) }\end{array}$ \\
\hline & 1018 & \pm & 185 & & & & 86 & \pm & 16 & & & & 185 & \pm & 24 & & \\
\hline 50 & 1888 & \pm & 314 & & & 20 & 170 & \pm & 26 & & & 20 & 84 & \pm & 7 & & \\
\hline 75 & 378 & \pm & 54 & & & 40 & 105 & \pm & 20 & & & 40 & 159 & \pm & 28 & & \\
\hline 99 & 174 & \pm & 29 & 13 & 5.0 & 60 & 112 & \pm & 21 & & & 60 & 103 & \pm & 18 & & \\
\hline 200 & 451 & \pm & 64 & 14 & 8.9 & 80 & 119 & \pm & 20 & & & 80 & 169 & \pm & 29 & & \\
\hline 400 & 433 & \pm & 63 & 46 & 26 & 100 & 155 & \pm & 26 & -0.1 & 2.3 & 100 & 227 & \pm & 37 & 16 & 14 \\
\hline 599 & 388 & \pm & 60 & 77 & 42 & 120 & 308 & \pm & 49 & & & 120 & 346 & \pm & 51 & & \\
\hline 800 & 396 & \pm & 61 & & & 140 & 294 & \pm & 51 & 13 & 10 & 140 & 472 & \pm & 68 & 17 & 14 \\
\hline \multirow[t]{10}{*}{1000} & 320 & \pm & 47 & 49 & 28 & 161 & 480 & \pm & 80 & & & 160 & 599 & \pm & 106 & & \\
\hline & & & & & & 200 & 646 & \pm & 93 & 13 & 10 & 180 & 590 & \pm & 100 & & \\
\hline & & & & & & 300 & 566 & \pm & 93 & 34 & 20 & 200 & 565 & \pm & 87 & 15 & 13 \\
\hline & & & & & & 380 & 489 & \pm & 79 & 33 & 19 & 300 & 711 & \pm & 106 & 16 & 13 \\
\hline & & & & & & 450 & 644 & \pm & 111 & 78 & 37 & 400 & 621 & \pm & 95 & 16 & 12 \\
\hline & & & & & & 500 & 386 & \pm & 58 & 71 & 35 & 500 & 388 & \pm & 65 & 41 & 23 \\
\hline & & & & & & 598 & 342 & \pm & 51 & 63 & 32 & 600 & 496 & \pm & 80 & & \\
\hline & & & & & & 700 & 293 & \pm & 50 & 56 & 28 & 700 & 644 & \pm & 103 & 62 & 32 \\
\hline & & & & & & 800 & 354 & \pm & 54 & 47 & 25 & 800 & 241 & \pm & 54 & 57 & 31 \\
\hline & & & & & & 1000 & 269 & \pm & 46 & 40 & 22 & 1000 & 202 & \pm & 48 & 48 & 29 \\
\hline
\end{tabular}




\begin{tabular}{|c|c|c|c|c|c|c|c|c|c|c|c|c|c|c|c|c|c|}
\hline \multicolumn{6}{|c|}{ Station $44\left(59.6^{\circ} \mathrm{N} /-38.9^{\circ} \mathrm{E}\right)$} & \multicolumn{6}{|c|}{ Station $51\left(59.8^{\circ} \mathrm{N} /-42.0^{\circ} \mathrm{E}\right)$} & \multicolumn{6}{|c|}{ Station $53\left(59.9^{\circ} \mathrm{N} /-43.01^{\circ} \mathrm{E}\right)$} \\
\hline \multirow{2}{*}{$\begin{array}{l}\text { Depth } \\
\text { (m) }\end{array}$} & \multirow{2}{*}{\multicolumn{3}{|c|}{$\begin{array}{c}\mathrm{Ba}_{\mathrm{xs}} \\
\left(\mathrm{pmol} \mathrm{L}^{-1}\right)\end{array}$}} & \multirow{3}{*}{$\begin{array}{l}\text { AOU } \\
(\mu \mathrm{mol} \\
\left.\mathrm{kg}^{-1}\right) \\
\end{array}$} & \multirow{2}{*}{$\begin{array}{l}\text { Water } \\
\text { mass age } \\
\text { (years) }\end{array}$} & \multirow{2}{*}{$\begin{array}{l}\text { Depth } \\
(\mathrm{m})\end{array}$} & \multirow{2}{*}{\multicolumn{3}{|c|}{$\begin{array}{c}\mathrm{Ba}_{\mathrm{xs}} \\
\left(\mathrm{pmol} \mathrm{L}{ }^{-1}\right)\end{array}$}} & \multirow{3}{*}{$\begin{array}{l}\text { AOU } \\
(\mu \mathrm{mol} \\
\left.\mathrm{kg}^{-1}\right) \\
\end{array}$} & \multirow{2}{*}{$\begin{array}{l}\text { Water } \\
\text { mass age } \\
\text { (years) }\end{array}$} & \multirow{2}{*}{$\begin{array}{l}\text { Depth } \\
\text { (m) }\end{array}$} & \multirow{2}{*}{\multicolumn{3}{|c|}{$\begin{array}{c}\mathrm{Ba}_{\mathrm{xs}} \\
\left(\mathrm{pmol} \mathrm{L}{ }^{-1}\right)\end{array}$}} & \multirow{3}{*}{$\begin{array}{l}\text { AOU } \\
(\mu \mathrm{mol} \\
\left.\mathrm{kg}^{-1}\right) \\
\end{array}$} & \multirow{2}{*}{$\begin{array}{c}\text { Water } \\
\text { mass age } \\
\text { (years) }\end{array}$} \\
\hline & & & & & & & & & & & & & & & & & \\
\hline 10 & 116 & \pm & 14 & & & 10 & 127 & \pm & 25 & & & 10 & 295 & \pm & 122 & & \\
\hline 20 & 90 & \pm & 7 & & & 19 & 61 & \pm & 8 & & & 20 & 203 & \pm & 133 & & \\
\hline 40 & 292 & \pm & 48 & & & 40 & 180 & \pm & 36 & & & 40 & 137 & \pm & 80 & & \\
\hline 60 & 293 & \pm & 46 & & & 60 & 194 & \pm & 37 & & & 60 & 98 & \pm & 75 & & \\
\hline 80 & 298 & \pm & 45 & & & 80 & 266 & \pm & 49 & & & 80 & 100 & \pm & 98 & & \\
\hline 100 & 464 & \pm & 68 & 18 & 11 & 100 & 300 & \pm & 54 & 5.6 & 6.1 & 100 & 218 & \pm & 114 & & \\
\hline 120 & 431 & \pm & 64 & & & 120 & 307 & \pm & 58 & & & 120 & 284 & \pm & 113 & & \\
\hline 140 & 501 & \pm & 83 & 19 & 12 & 140 & 316 & \pm & 59 & 15 & 8.7 & 140 & 129 & \pm & 143 & & \\
\hline 160 & 605 & \pm & 91 & & & 160 & 359 & \pm & 69 & & & & & & & & \\
\hline 200 & 695 & \pm & 104 & 18 & 11 & 180 & 251 & \pm & 55 & & & & & & & & \\
\hline 300 & 739 & \pm & 116 & 18 & 13 & 200 & 383 & \pm & 72 & 18 & 10 & & & & & & \\
\hline 400 & 747 & \pm & 111 & 19 & 12 & 300 & 495 & \pm & 84 & 22 & 12 & & & & & & \\
\hline 500 & 653 & \pm & 106 & 20 & 13 & 400 & 445 & \pm & 78 & 29 & 13 & & & & & & \\
\hline 600 & 483 & \pm & 83 & 24 & 15 & 500 & 299 & \pm & 65 & 38 & 17 & & & & & & \\
\hline 700 & 823 & \pm & 134 & 25 & 15 & 600 & 278 & \pm & 46 & & & & & & & & \\
\hline 800 & 632 & \pm & 89 & 22 & 14 & 700 & 275 & \pm & 47 & & & & & & & & \\
\hline 1100 & 284 & \pm & 54 & 40 & 24 & 800 & 225 & \pm & 47 & 40 & 17 & & & & & & \\
\hline \multirow[t]{2}{*}{1401} & 298 & \pm & 52 & & & 1000 & 199 & \pm & 34 & 38 & 27 & & & & & & \\
\hline & \multicolumn{5}{|c|}{ Station $64\left(59.1^{\circ} \mathrm{N} /-46.1^{\circ} \mathrm{E}\right)$} & \multicolumn{6}{|c|}{ Station $69\left(55.8^{\circ} \mathrm{N} /-48.1^{\circ} \mathrm{E}\right)$} & \multicolumn{6}{|c|}{ Station $77\left(53.0^{\circ} \mathrm{N} /-51.1^{\circ} \mathrm{E}\right)$} \\
\hline Depth & & $\mathrm{Ba}_{\mathrm{xs}}$ & & AOU & $\begin{array}{c}\text { Water } \\
\text { mass age }\end{array}$ & & & $\mathrm{Ba}_{\mathrm{xs}}$ & & & $\begin{array}{c}\text { Water } \\
\text { mass age }\end{array}$ & & & $\mathrm{Ba}_{\mathrm{xs}}$ & & & $\begin{array}{c}\text { Water } \\
\text { mass age }\end{array}$ \\
\hline$(\mathrm{m})$ & & mol L & & $\begin{array}{c}(\mu \mathrm{mol} \\
\left.\mathrm{kg}^{-1}\right)\end{array}$ & (years) & $(\mathrm{m})$ & & nol & & $\begin{array}{c}(\mu \mathrm{mol} \\
\left.\mathrm{kg}^{-1}\right)\end{array}$ & (years) & $(\mathrm{m})$ & & nol L & & $\begin{array}{c}(\mu \mathrm{mol} \\
\left.\mathrm{kg}^{-1}\right)\end{array}$ & (years) \\
\hline 10 & 61 & \pm & 13 & & & 11 & 58 & \pm & 13 & & & 10 & 104 & \pm & 6 & & \\
\hline 20 & 143 & \pm & 32 & & & 20 & 148 & \pm & 11 & & & 20 & 113 & \pm & 9 & & \\
\hline 40 & 99 & \pm & 22 & & & 41 & 178 & \pm & 40 & & & 39 & 170 & \pm & 16 & & \\
\hline 60 & 97 & \pm & 22 & & & 60 & 198 & \pm & 46 & & & 60 & 316 & \pm & 57 & & \\
\hline 80 & 112 & \pm & 23 & & & 80 & 358 & \pm & 72 & & & 80 & 374 & \pm & 63 & & \\
\hline 100 & 282 & \pm & 47 & 10 & 7.3 & 100 & 459 & \pm & 89 & 20 & 13 & 100 & 411 & \pm & 71 & 6.8 & 7.8 \\
\hline 120 & 432 & \pm & 77 & & & 120 & 505 & \pm & 96 & & & 120 & 381 & \pm & 68 & & \\
\hline 140 & 506 & \pm & 86 & 17 & 9.0 & 140 & 550 & \pm & 102 & 22 & 14 & 140 & 369 & \pm & 61 & 11 & 9.6 \\
\hline 160 & 458 & \pm & 91 & & & 160 & 525 & \pm & 91 & & & 160 & 418 & \pm & 84 & & \\
\hline 199 & 447 & \pm & 89 & 19 & 10 & 200 & 619 & \pm & 97 & 20 & 13 & 200 & 493 & \pm & 87 & 15 & 11 \\
\hline 300 & 500 & \pm & 104 & 19 & 12 & 300 & 768 & \pm & 121 & 23 & 13 & 300 & 503 & \pm & 75 & 17 & 12 \\
\hline 400 & 332 & \pm & 82 & 20 & 12 & 400 & 682 & \pm & 119 & 22 & 14 & 401 & 470 & \pm & 80 & 23 & 13 \\
\hline 500 & 717 & \pm & 115 & 18 & 10 & 502 & 778 & \pm & 123 & 23 & 13 & 500 & 509 & \pm & 86 & 23 & 16 \\
\hline 600 & 696 & \pm & 100 & & & 601 & 831 & \pm & 135 & 23 & 15 & 601 & 445 & \pm & 73 & 24 & 14 \\
\hline 700 & 516 & \pm & 83 & 22 & 13 & 700 & 863 & \pm & 135 & & & 700 & 561 & \pm & 88 & 24 & 15 \\
\hline 800 & 750 & \pm & 121 & 19 & 11 & 802 & 744 & \pm & 113 & 23 & 15 & 800 & 575 & \pm & 101 & 26 & 16 \\
\hline 900 & 630 & \pm & 108 & 22 & 14 & 900 & 663 & \pm & 111 & & & 901 & 583 & \pm & 93 & 25 & 15 \\
\hline 1000 & 580 & \pm & 98 & 25 & 15 & 1001 & 741 & \pm & 119 & 24 & 16 & 1002 & 475 & \pm & 70 & 25 & 15 \\
\hline & & & & & & 1500 & 535 & \pm & 91 & & & & & & & & \\
\hline
\end{tabular}

